NASA Technical Memorandum 103259

AIAA-90-2669

\title{
Geometric Effects in Applied-Field MPD Thrusters
}

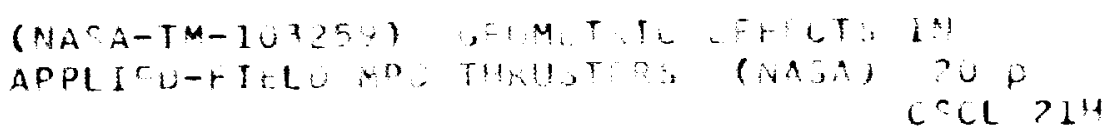

$12 u-277 ?$

R.M. Myers

Sverdrup Technology, Inc.

Lewis Research Center Group

Brook Park, Ohio

and

M. Mantenieks and J. Sovey

National Aeronautics and Space Administration

Lewis Research Center

Cleveland, Ohio

Prepared for the

21st International Electric Propulsion Conference

sponsored by AIAA/DGLR/JSASS

Orlando, Florida, July 18-20, 1990 



\title{
Geometric Effects in Applied-Field MPD Thrusters
}

\author{
R.M. Myers \\ Sverdrup Technology, Inc. \\ Lewis Research Center Group \\ Brook Park, Ohio 44142 \\ M. Mantenieks and J. Sovey \\ National Aeronautics and Space Administration \\ Lewis Research Center \\ Cleveland, Ohio 44135
}

\begin{abstract}
Three applied-field MPD thruster geometries were tested with argon propellant to establish the influence of electrode geomety on thruster performance. The thrust increased approximately linearly with anode radius, while the discharge and electrode fall voltages increased quadratically with anode radius. All these parameters increased linearly with applied-field strength. Thrust efficiency, on the other hand, was not significantly influenced by changes in geometry over the operating range studied, though both thrust and thermal efficiencies increased monotonically with applied field strength. The best performance, $1820 \mathrm{sec} \mathrm{I}_{\mathrm{sp}}$ at $20 \%$ efficiency, was obtained with the largest radius anode at the highest discharge current (1500 amps) and applied field strength (0.4 Tesla).
\end{abstract}

\section{Nomenclature}

\begin{tabular}{|c|c|c|}
\hline $\begin{array}{l}B \\
D_{e} \\
e \\
E\end{array}$ & - & $\begin{array}{l}\text { applied mitgnetic field strength, } \mathrm{T} \\
\text { electron diffusion coefficient } \\
\text { electron charge, } \mathrm{C} \\
\text { electric field }\end{array}$ \\
\hline $\begin{array}{l}\mathrm{je}_{\mathrm{e}} \\
\mathrm{J}_{\mathrm{d}} \\
\mathrm{k}\end{array}$ & - & $\begin{array}{l}\text { electron current density, } \mathrm{A} / \mathrm{cm}^{2} \\
\text { discharge current, } \mathrm{A} \\
\text { Boltzmann's constant, } \mathrm{J} / \mathrm{K}\end{array}$ \\
\hline $\ln (\Lambda)$ & - & Coulomb logarithm \\
\hline $\mathrm{m}$ & - & mass flow rate, $\mathrm{g} / \mathrm{s}$ \\
\hline $\mathrm{m}_{\mathrm{e}}, \mathrm{n}_{\mathrm{e}}$ & - & electron mass, $\mathrm{kg}$, and number density, $\mathrm{cm}^{-3}$ \\
\hline$r_{a}, r_{c}$ & - & anode and cathode radii, $\mathrm{cm}$ \\
\hline $\mathrm{r}_{1}$ & - & electron Larmor radius, $\mathrm{cm}$ \\
\hline $\mathrm{T}$ & - & thrust, $\mathrm{N}$ \\
\hline $\mathrm{T}_{\mathrm{e}}$ & - & electron temperature, $\mathrm{eV}$ \\
\hline $\mathrm{U}_{\mathrm{c}}$ & - & Alfven critical ionization velocity, $\mathrm{m} / \mathrm{s}$ \\
\hline $\mathrm{V}_{\mathrm{d}}, \mathrm{V}_{\mathrm{e}}$ & - & discharge and electrode fall voltages, $\mathrm{V}$ \\
\hline y & - & linear distance \\
\hline$\lambda_{d}$ & - & Debye length, $\mathrm{cm}$ \\
\hline$\eta_{t}, \eta_{t h}$ & - & thrust and thermal efficiencies \\
\hline$\Gamma_{\mathrm{e}}$ & - & electron number flux \\
\hline$\mu_{\mathrm{e}}, \mu_{\mathrm{o}}$ & - & electron mobility and permeability of free space \\
\hline$\omega_{\mathrm{ce}}$ & - & electron cyclotron frequency, $\mathrm{s}^{-1}$ \\
\hline$v_{e-i}$ & - & electron - ion collision frequency, $\mathrm{s}^{-1}$ \\
\hline
\end{tabular}




\section{Introduction}

Analyses of lunar and Mars missions utilizing nuclear electric propulsion systems have shown that the thruster specific impulse should be between 5000 and 8000 seconds with an efficiency over 50 percent $^{1}$. In addition, the propulsion system must deliver a total impulse of approximately $1 \times 10^{9} \mathrm{~N}$-s while operating at average power levels between 2 and $10 \mathrm{MW} 1,2$. While MPD thrusters may be able to fulfill these requirements, the large disparities between the mission requirements and the capabilities of current technology have forced a multi-faceted approach to address these issues, including direct measurements in quasi-steady thrusters at multi-megawatt power levels and scaled testing of steady-state thrusters at lower powers. This paper presents preliminary results of one aspect of the research directed at establishing the feasibility and practicality of MPD thruster technology.

The highest efficiencies and specific impulses are reported for multi-megawatt quasi-steady MPD thrusters with applied magnetic fields using hydrogen propellant, with reported values reaching $70 \%$ at 7000 seconds $\mathrm{I}_{\mathrm{sp}}{ }^{3}$. Quasi-steady self-field thruster efficiency is generally lower ${ }^{3-5}$. Thrusters utilizing other propellants $\left(\mathrm{Ar}, \mathrm{He}, \mathrm{NH}_{3}, \mathrm{~N}_{2}\right.$, and $\mathrm{Ne}$ ) have still lower performance, with peak values of approximately $30-40 \%$ and specific impulses up to 4000 seconds $^{2}$. Results for steady-state thrusters operated at powers up to $600 \mathrm{~kW}$ cover a similarly wide range, with the highest performance reported with an applied field thruster operated on lithium at powers below $30 \mathrm{~kW}\left(7000 \mathrm{sec} \mathrm{I}_{\mathrm{sp}}\right.$, $60 \%$ efficiency 6 ). Self-field thruster testing at powers up to $600 \mathrm{~kW}$ has been done with argon 7 , with a maximum performance of less than $25 \%$ efficiency at 1500 seconds $\mathrm{I}_{\mathrm{sp}}$.

Results to date have been obtained with a broad range of thruster geometries, with power levels ranging from tens to thousands of kilowatts, propellant flow rates from 0.005 to $10 \mathrm{~g} / \mathrm{s}$, and a variety of propellants. The very range of the MPD thruster research makes it at present difficult to conclusively establish the performance trends for either self or applied-field thrusters. This problem is compounded by the lack of detailed theoretical models to guide the design of more efficient thrusters. Previous modelling efforts for both self-field ${ }^{8,9}$ and applied-field ${ }^{10-12}$ thrusters have shown substantial promise, but they have not been verified or implemented for a variety of thruster conditions to indicate directions for research. For example, the importance of the shape and strength of the magnetic field or the electrode size and geometry are not presently understood. The combined lack of an extensive database of directly measured performance and detailed models has precluded significant advances in MPD thruster performance.

This paper presents preliminary results of an effort to systematically study the impact of geometry and operating condition on MPD thruster performance. Following a description of the test facility and the thruster geometries, a summary of the performance measurements will be given, including measurements of power lost to the electrodes. Simple models are then used to interpret the observed trends.

\section{Experimental Apparatus}

\section{Test Facility}

The MPD thrusters were mounted on a thrust stand in a $3 \mathrm{~m}$ diameter, $3 \mathrm{~m}$ long test section of a $7.6 \mathrm{~m}$ diameter, $21 \mathrm{~m}$ long main vacuum chamber. The $3 \mathrm{~m}$ diameter gate valve between the test section and the main tank was opened after inserting the thrust stand into the test section. The test stand is shown in Fig. 1. The tank pressure was maintained 
below $0.07 \mathrm{~Pa}$ during testing at argon flow rates up to $0.14 \mathrm{~g} / \mathrm{s}$. Thruster power was provided by a series-parallel network of $66 \mathrm{~kW}$ welding supplies with a maximum capability of $3000 \mathrm{~A}$ at $88 \mathrm{~V}$. The propellant for these tests was argon. Propellant flow rates and thrust levels were calibrated to within $5 \%$ and $2 \%$, respectively. In-situ thrust calibrations were done using a motor driven weight suspension apparatus. Details of the thrust stand and its calibration, tares, etc., are given in Ref. 13.

The thruster discharge was initiated using a combination of a $1 \mathrm{kV}, 2$ amp power supply connected across the thruster electrodes and a short duration gas pulse to temporarily raise the thruster chamber pressure. The main power supplies were protected from the high voltage pulse using blocking diodes in series with the supplies. Generally a 1 - 2 second gas pulse followed by a 0.1 second voltage pulse was sufficient to ignite the discharge. A short current overshoot, lasting 1 - 3 seconds, preceded stabilization at the preset current level.

Water cooling for the thruster and applied-field magnet was provided by two closed-loop heat exchangers. The water pumps supplied up to $0.8 \mathrm{l} / \mathrm{sec}$ at a pressure of $1 \mathrm{MPa}$. Water flow rates were measured using turbine flow meters calibrated to within $2 \%$. Further descriptions of the facility are found in References 13-15.

\section{MPD thrusters and Applied-Field Magnet}

The MPD thruster geometries tested in this work are shown in Fig. 2. These coaxial thrusters consisted of a central $1.27 \mathrm{~cm}$ diameter, $2 \%$ thoriated tungsten cathode with an annular copper anode. The anodes were water cooled along their entire length, while the cathodes were water cooled behind the thruster backplate. Two cylindrical anodes and one flared anode were tested in this work. "To permit comparisons between geometries the "effective anode radius" of the flared anode was defined as the average of the upstream and exit plane radii. While this appears arbitrary given our limited knowledge of the current distribution, reasons for the choice will become clear in the next sections. Thruster dimensions are given in Table 1. Geometries A and B were modified versions of commercially available plasma sprayers. These designs used O-ring seals, which failed at high input powers. These rubber seals were eliminated in geometry $\mathrm{C}$, which extended the thruster power range.

The applied-field magnet consisted of a solenoid made from 46 turns of water cooled copper tubing ( $1.27 \mathrm{~cm}$ O.D, $0.89 \mathrm{~mm}$ wall ) in a $14 \mathrm{~cm}$ ID, $15 \mathrm{~cm}$ long spool. The magnet had four layers of ten coils each, and a final layer with six coils. Thrusters were placed in the magnet such that the thruster exit plane was aligned with the magnet face to reduce the heat transfer to the solenoid from the thruster plume. The magnetic field at the magnet center reached a maximum of 0.4 Tesla with 1500 amps through the solenoid. A typical plot of axial applied-field strength vs. axial position along the centerline is given in Fig. 3. The axial field strength at the thruster exit plane is plotted vs. radius in Fig. 4. In this paper, the axial field strength at the centerline of the thruster exit plane is used as the reference value.

\section{Experimental Results}

The discharge voltage and thrust for the three thruster geometries, operated at a discharge current of 1000 amps and an argon flow rate of $0.14 \mathrm{~g} / \mathrm{s}$, are plotted versus the applied magnetic field strength (at the centerline of the thruster exit plane) in Figures 5 and 6 , respectively. The discharge voltage increased approximately linearly with applied-field

strength for all geometries. Linear least-squares fits to these data showed that for the two 
cylindrical geometries the slope of the discharge voltage versus applied field strength curve increased quadratically with the anode radius, while the flared anode has a lower slope than that implied by this relationship. The curve-fit results are summarized in Table 2 . For example, the slope for geometry $A$, with a radius of $1.3 \mathrm{~cm}$, was $37.2 \mathrm{~V} / \mathrm{Tesla}$, while for geometry $\mathrm{C}$, with a radius twice that of geometry $\mathrm{A}$, this slope was $149.8 \mathrm{~V} / \mathrm{Tesla}$, or four times the slope observed with geometry $A$. This scaling relationship will be discussed further in the next section. No clear correlations were found between the discharge voltage slope and the anode or cathode length, or with the discharge current.

As shown in Fig. 6, the thrust level also increased approximately linearly with applied-field strength for all geometries, and the slope is again strongly dependent on anode radius. As seen from the values in Table 3, however, the quantitative relationship between the slope and the anode radius is not as apparent. To further study this geometric effect, two tests were conducted with the cylindrical geometries (A and $\mathrm{C}$ ) in which both the discharge current and the applied-field strength were varied. As shown in Fig. 7, the thrust increased linearly with the product of the discharge current and the applied-field strength $\left(\mathrm{J}_{\mathrm{d}} \times \mathrm{B}\right)$ for both geometries. In addition, the data in Figure 7 show that the slope of the thrust- $\mathrm{J}_{\mathrm{d}} \times \mathrm{B}$ curve is directly proportional to the anode radius.

The thrust measurements resulted in the specific impulse values, defined by

$$
\mathrm{I}_{\mathrm{sp}}=\frac{\mathrm{T}}{\dot{\mathrm{m} g}}
$$

shown in Fig. 8. The maximum value of 1820 seconds was reached with the largest diameter anode at the highest discharge current and magnetic field strength.

The thrust efficiencies, defined by

$$
\eta_{\mathrm{T}}=\frac{\mathrm{T}^{2}}{2 \dot{\mathrm{m}} \mathrm{V}_{\mathrm{d}} \mathrm{J}_{\mathrm{d}}}
$$

are shown in Fig. 9 for a discharge current of $1000 \mathrm{~A}$ and argon flow rate of $0.14 \mathrm{~g} / \mathrm{s}$. The efficiencies for all three geometries were comparable, though at low applied-field strengths the smallest anode had a significantly higher efficiency. The efficiencies approach $20 \%$ for all geometries at high field strengths. It is clear that the efficiency of the larger anode (geometry $C$ ) depended more strongly on field strength than the smaller anodes (geometries A and B). In addition, the leveling off in the efficiency vs. applied field curve observed for the smallest anode (geometry A) was not seen with the larger anodes.

The principal cause of the low thrust efficiencies was electrode power deposition. The fraction of the total power deposited into the electrodes was established using calorimetric measurements of the water cooling the electrodes. These measurements were used to establish the thermal efficiency, defined by

$$
\eta_{\mathrm{th}}=1-\frac{\text { Electrode Power }}{\mathrm{V}_{\mathrm{d}} \mathrm{J}_{\mathrm{d}}}
$$

which is the fraction of the input power going into the plasma. The water flow rate to the electrode cooling passages was adjusted to maintain a minimum of six degrees Celsius 
temperature change between inlet and outlet. This ensured electrode power measurements to better than $10 \%$ with a thermocouple accuracy of 0.56 degrees Celsius.

Results of these measurements are shown in Fig. 10, where the thermal efficiency is plotted vs. the applied field strength. The thermal efficiencies for the two smaller radius thrusters are only weakly dependent on the magnetic field strength, and there is substantial scatter in the data for geometry A. Geometry C, with the largest anode radius, showed the strongest dependence on applied field strength, with values increasing from $25 \%$ at $0.1 \mathrm{~T}$ applied-field to $40 \%$ at $0.4 \mathrm{~T}$. It is clear that for all geometries over $50 \%$ of the input power was deposited into the electrodes.

The influence of the magnetic field on electrode power deposition is more clearly shown using an "effective electrode fall voltage", defined as the total power deposited into the electrodes divided by the discharge current. Results for the three geometries, shown in Figure 11 , indicate a linear rise in the effective electrode fall with magnetic field. The smallest geometry has both the lowest magnitude electrode fall and the slowest rise with applied-field strength. The highest value, 44 volts, is reached with the largest thruster (geometry C) at the highest field strength. Linear least-squares fits for the two cylindrical geometries, summarized in Table 4 , indicate that the slopes increased quadratically with the anode radius. Once again, using the effective radius for the flared anode thruster yields a slope too high for this relationship.

\section{Discussion}

The above data have several implications. First, since thrust is approximately proportional to the anode radius, and the discharge voltage is proportional to radius squared, the thrust efficiency should be approximately independent of anode radius, as observed. However, the thermal efficiencies for the three geometries were significantly different for low applied-field strengths, with the larger anode thruster losing more power to the electrodes. This indicates that the larger anodes must recover more of the energy deposited into the plasma. Thus, while the overall power balance does not appear to change, the relative magnitudes of the power sinks do.

The measured values of specific impulse, while low when compared to mission requirements, are much higher than expected for simple self-field acceleration. Using the standard self-field thrust equation with an effective anode to cathode radius ratio 16

$$
\mathrm{T}=\frac{\mu_{0} J_{d}^{2}}{4 \pi} \ln \left(\frac{r_{a}}{r_{c}}\right)_{\text {eff }}
$$

for the geometries tested yields specific impulses of 52,75 , and 102 seconds, respectively, for the test conditions. These values are orders-of-magnitude below those measured. While at low applied-field strengths thermal effects may be important with the small anode geometries, the high specific impulses observed for high applied-fields cannot be explained by thermal acceleration of argon. It is clear that the dominant plasma acceleration was due to the applied-field.

The theoretical model that most clearly lends itself to interpreting the above measurements is that of Fradkin ${ }^{10}$. He assumed that the applied-field acceleration was dominant, that rotational motion was converted to axial motion with constant efficiency, and that the plasma followed a magnetic field flux tube, and solved the equations of 
continuity and conservation of angular momentum and energy to establish the parametric behavior of applied-field MPD thrusters. Fradkin experimented with only one thruster geometry, and so was not able to verify the model predictions with regards to anode shape. While highly simplified, some of the general trends predicted by the model agree with those observed in this work. Specifically, Fradkin predicted that the electromagnetic thrust and discharge voltage scale as

and

$$
\mathrm{T}=\frac{1}{\sqrt{2}} \mathrm{BJ}_{\mathrm{d}} \mathrm{r}_{\mathrm{a}}\left[1-\frac{3}{2}\left(\frac{\mathrm{r}_{\mathrm{c}}}{\mathrm{r}_{\mathrm{a}}}\right)^{2}\right]
$$

$$
V_{d}=V_{c}+\frac{B^{2} J_{d} r_{a}^{2}}{2 \dot{m}}
$$

where $V_{c}$ is the voltage due to ohmic heating and the electrode falls. The thrust scaling arises from the dependence of the applied-field torque on the product of the field strength and the discharge current. Note that for the geometries studied, the second term in the brackets is small. The linear increase of thrust with anode radius is due to the linear decrease in current density with anode radius and the quadratic increase in cross-sectional area. The voltage scaling arises from the back-emf $(\mathrm{UxB})$ term in Ohm's Law: the velocity is proportional to $B J_{d r_{a}}$ (from thrust), so the product $U x B$ scales as $B^{2} J_{d} r_{a}$. The second power of the radius arises from the linear increase in path length with anode radius.

The linear increase in thrust with $\mathrm{BJ}_{\mathrm{d}} \mathrm{r}_{\mathrm{a}}$ matches the results shown in Figure 7. However, while the discharge voltage plotted in Figure 5 does increase quadratically with anode radius, it does not appear to increase quadratically with the magnetic field strength and testing revealed that it is not sensitive to the discharge current. This same discrepancy was observed by Fradkin, who attempted to explain it by invoking the Alfven critical velocity hypothesis. For a velocity limited case, the voltage should scale as

$$
V_{d}=V_{c}+U_{c} B r_{a}\left(1-\frac{r_{c}^{2}}{r_{a}^{2}}\right)
$$

where $U_{c}$ is the Alfven critical velocity. This has the same behavior as our measurements except for the radius dependence. Thus, while the model correctly predicts thrust scaling behavior, there is a problem with the voltage prediction which must be resolved by future models.

The discrepancy between the geometry scaling for the cylindrical anodes and that for the flared anode is most probably due to a non-uniform current distribution in the latter geometry. To substantiate this conclusion, the "true effective anode radius" for the flared anode (geometry B) was calculated assuming the scaling laws of $V \sim r_{a}^{2}, T \sim r_{a}$ and $V_{e} \sim$ $r_{a}{ }^{2}$, and using the measured slopes to calculate $r_{a}$. The results were uniformly smaller than the geometric average radius by between two and three millimeters, indicating that the current attached preferentially on the upstream side of the anode in the flared anode thruster.

The linear increase in the effective electrode fall voltage with applied magnetic field indicates that the sheath is magnetized. This occurs whenever the electron gyro radius is comparable to or smaller than the Debye length, and the electron cyclotron frequency is 
much larger than the electron- ion collision frequency. These ratios are calculated from ${ }^{17}$

and

$$
\frac{r_{1}}{\lambda_{d}}=\frac{7.5 \times 10^{-11}}{\mathrm{eB}} \sqrt{\mathrm{m}_{\mathrm{e}} \mathrm{n}_{\mathrm{c}}}
$$

$$
\frac{\omega_{c e}}{v_{e-i}}=\frac{\mathrm{eBT}_{\mathrm{e}}^{1.5}}{2 \times 10^{-6} \mathrm{~m}_{\mathrm{e}} \mathrm{n}_{\mathrm{e}} \ln (\Lambda)}
$$

Note that the first relation is independent of temperature. Values for argon at several magnetic fields and electron densities are given in Table 5. The Coulomb logarithm and the ion charge were given constant values of ten and one (singly ionized), respectively, for these estimates, so the results should be within a factor of two of actual values. The electron densities bracket measurements made using electrostatic probes close to the thruster exhaust plane ${ }^{18}$. It is clear that for most of the operating conditions studied here the electron Larmor radius is comparable to the Debye length and that electrons cycle about magnetic field lines many times before undergoing a collision. These estimates substantiate the conclusion that the magnetic field impedes electron motion in the sheath, and they have important implications for methods used to model electrode processes in applied-field thrusters.

The linear rise in effective fall voltage with applied-field strength can be explained in part by considering the diffusion of electrons into the anode. Previous studies of steadystate thrusters have concluded that the anode fall is the dominant source of electrode power deposition, so that the data presented above are most likely representative of processes occuring near the anode ${ }^{19-21}$. The electron flux into the anode can be written as 22

$$
\Gamma_{e}=-D_{e} \frac{d n_{e}}{d y}-\mu_{e} n_{e} \vec{E}
$$

Using the Bohm diffusion coefficient ${ }^{17}$ for $\mathrm{D}_{\mathrm{e}}$ and rearranging using Einstein's relation between the diffusion crefficient and the electron mobility, we obtain

$$
\vec{E}=-\frac{1}{e n_{e}}\left(16 j_{e} B+k T_{e} \frac{d n_{c}}{d y}\right)
$$

which shows that, to a first order, the electric field required to maintain a constant electron current increases linearly with magnetic field strength. Note that classical diffusion would predict a quadratic increase in electric field with magnetic field strength, a dependence which is clearly not observed in these measurements. Limitations of this argument include its neglect of ion current and our lack of knowledge of how the other terms vary with magnetic field strength.

\section{Conclusions}

Prelimary studies of the influence of applied-field thruster geometry on performance showed that the anode radius had a large effect on thrust, discharge voltage and electrode fall voltage for a given operating point. Specifically, thrust appeared to increased linearly 
with anode radius, and the discharge and electrode fall voltages increased quadradically with anode radius. These quantities increased linearly with applied field strength over the operating range covered in this study, and thrust was proportional to the product of the applied field strength and the discharge current. These effects are such that the thrust efficiency is not significantly influenced by thruster geometry, though both thermal and thrust efficiency increased monotonically with magnetic field strength. The results show that while thruster geometry did not dramatically affect the overall thruster power balance, the magnitudes of the dominant power sinks changed with geometry. It is important to note that the operating range studied here did not include regions where self-field effects contribute significantly to plasma acceleration.

\section{Acknowledgements}

The authors would like to thank Larry Schultz and the Tank 6 mechanical crew, Gerry Schneider and Rob Buttler for their assistance in preparing and maintaining the experimental apparatus.

\section{References}

1. Palaszewski, B., "Electric Propulsion Parameters for Manned Mars Exploration," JANNAF Prop. Meeting, May 1989

2. Sovey, J. and Mantenieks, M., "Performance and Lifetime Assessment of MPD Arc Thruster Technology," AIAA 88-3211 (NASA TM 101293), July 1988

3. Tahara, H., Kagaya, Y., and Yoshikawa, T., "Hybrid MPD Thruster with Axial and Cusp Magnetic Fields," IEPC 88-058, Oct. 1988

4. Tahara, H., et al, "Development of a Quasi-Steady MPD Arcjet Thruster for Near-Earth Missions," AIAA 87-1001, May 1987

5. Kagaya, Y., Yoshikawa, T., and Tahara, H., "Quasi-Steady MPD Arcjets with Applied Magnetic Fields," AIAA 85-2001, Oct. 1985

6. Connolly, D. J., et al., "Low Environmental Pressure MPD Arc Tests," ALAA J., Vol. 6, No. 7, July 1968, pp. 1271-1276

7. Merke, W., et al., "Nozzle Type MPD Thruster Experimental Investigations," IEPC 88028, Oct. 1988

8. Auweter-Kurtz, M., et al., "An Improved Code for Nozzle Type Steady-State MPD Thrusters," IEPC 88-040, Oct. 1988

9. Martinez-Sanchez, M., "The Structure of Self-Accelerated Plasma Flows," AIAA 87 1065, May 1987

10. Fradkin, D., "Analysis of Acceleration Mechanisms and Performance of an Applied Field MPD Arcjet," Ph.D Thesis, Princeton University, March 1973

11. Kruelle, G., "Theoretical Treatment of Current, Mass Flow, and Related Distributions in MPD Plumes," AIAA 72-501, April 1972

12. Tanaka, M. and Kimura, I., "Current Distribution and Plasma Acceleration in MPD Arcjets with Applied Magnetic Fields," L. Prop_and Power, Vol. 4, No. 5, Sept.-Oct. 1988, pp. 428-436

13. Haag, T. W., "Design of Thrust Stand for High Power Electric Propulsion Devices," AIAA-89-2829, July 1989

14. Sovey, J. S., et al., "Test Facility and Preliminary Performance of a $100 \mathrm{~kW}$ Class MPD Thruster," NASA TM 102021, May 1989

15. Mantenieks, M., et al., "Performance of a $100 \mathrm{~kW}$ Class Applied Field MPD Thruster," AIAA-89-2710 (NASA TM 102312), July 1989 
16. Gilland, J. "Influence of Geometric Scale upon MPD Thruster Performance," M.S. Thesis, Princeton University, March, 1988

17. Chen, F., Introduction to Plasma Physics and Controlled Fusion, Vol. 1, Plenum Press, New York, 1985

18. Myers, R. M., "Plume Characteristics of MPD Thrusters: A Preliminary Examination," AIAA-89-2832, July 1989

19. Myers, R. M., Kelly, A.J., and Jahn, R.G., "Energy Deposition in Low-Power Coaxial Plasma Thrusters," IEPC 88-024, Oct. 1988

20. Oberth, R. and Jahn, R., "Anode Phenomena in High-Current Accelerators," AIAA J., Vol. 10, No. 1, Jan 1972

21. Shih, K., et al., "Experimental Studies of the Electrode Heat Transfer in a MPD Arc Configuration," AIAA 67-673, July 1967

22. Kalikhman, L. , Elements of Magnetogasdynamics, trans. by Scripta Technica, Inc.,

W. B. Saunders Co., Philadelphia, 1967 
Table 1: MPD thruster dimensions

\begin{tabular}{cccc} 
Geomerry & anode radius $(\mathrm{cm})$ & Anode Length $(\mathrm{cm})$ & Cathode Length $(\mathrm{cm})$ \\
\hline A & 1.3 & 4.2 & 2.2 \\
B & 1.8 (effective) & 5.4 & 2.2 \\
C & 2.6 & 7.6 & 7.6
\end{tabular}

\begin{tabular}{cccc} 
& Table 2: & Discharge voltage slopes and intercepts \\
\cline { 2 - 4 } Geometry & Anode Radius (cm) & Slope (Volis/Tesla) & Y intercept (Volts) \\
\hline A & 1.3 & 37.2 & 16.4 \\
B & 1.8 (effective) & 54 & 22.2 \\
C & 2.6 & 149.8 & 10.6
\end{tabular}

\begin{tabular}{|c|c|c|c|}
\hline Geometry & Anode Radius (cm) & Slope (Newtons/Tesla) & Y intercepl (Newtons) \\
\hline $\mathbf{A}$ & 1.3 & 1.7 & 0.7 \\
\hline B & 1.8 (effective) & 2.2 & 0.8 \\
\hline $\mathrm{C}$ & 2.6 & 4.1 & 0.3 \\
\hline
\end{tabular}

Table 4: Effective electrode fall slopes and intercepts

\begin{tabular}{cccc} 
Geometry & Anode Radius (cm) & Slope (Volts/Tesla) & Intercept (Volts) \\
\hline A & 1.3 & 18.1 & 10.3 \\
B & 1.8 (effective) & 23 & 15.1 \\
C & 2.6 & 73 & 12.7
\end{tabular}


Table 5: Ratio of Larmor radius to Debye length and gyro frequency to electron-ion collision frequency

\begin{tabular}{cccrc}
$\mathrm{B}$ (Tesla) & $\mathrm{n}_{\mathrm{e}}\left(\mathrm{cm}^{-3}\right)$ & $\mathrm{T}_{\mathrm{e}}(\mathrm{eV})$ & $\mathrm{r}_{1} \lambda_{d}$ & $\omega_{\mathrm{ce}} N_{\mathrm{e}-\mathrm{i}}$ \\
\hline 0.1 & $10^{11}$ & 1 & 1.4 & $8.8 \times 10^{3}$ \\
0.1 & $10^{13}$ & 1 & 14.1 & $8.8 \times 10^{1}$ \\
0.1 & $10^{11}$ & 5 & 1.5 & $9.8 \times 10^{4}$ \\
0.1 & $10^{13}$ & 5 & 14.1 & $9.8 \times 10^{2}$ \\
0.4 & $10^{11}$ & 1 & 0.4 & $3.5 \times 10^{4}$ \\
0.4 & $10^{13}$ & 1 & 3.5 & $3.5 \times 10^{2}$ \\
0.4 & $10^{11}$ & 5 & 0.4 & $3.9 \times 10^{5}$ \\
0.4 & $10^{13}$ & 5 & 3.5 & $3.9 \times 10^{3}$
\end{tabular}




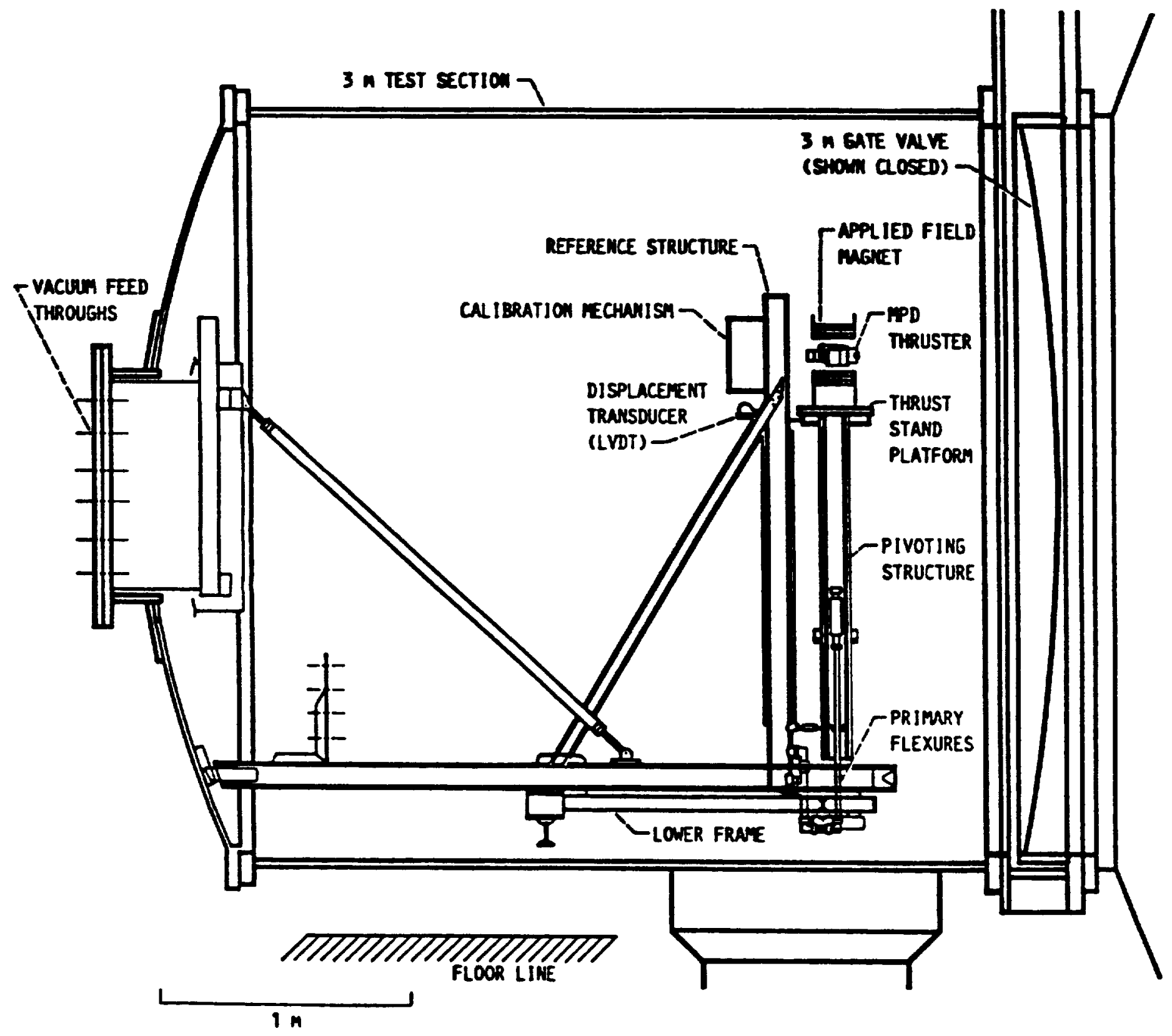

Figure 1: MPD Thruster Test Stand 


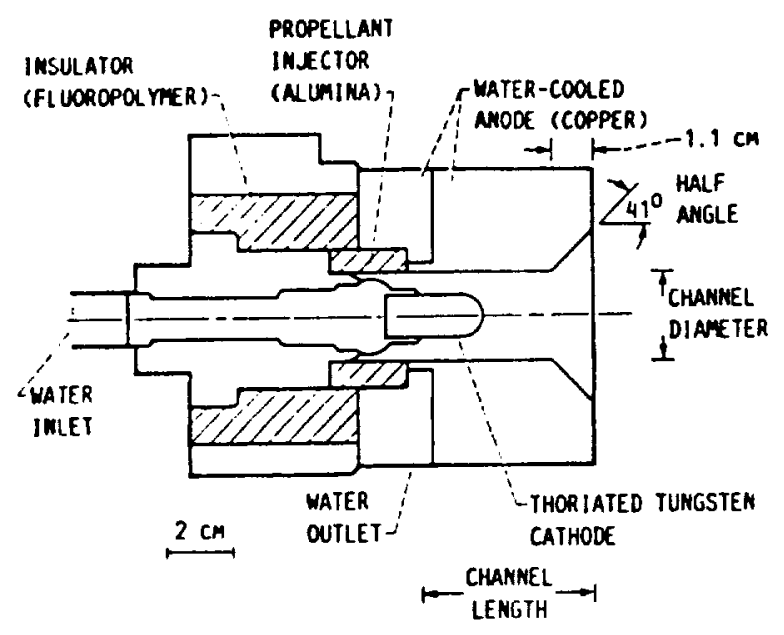

Geometry A

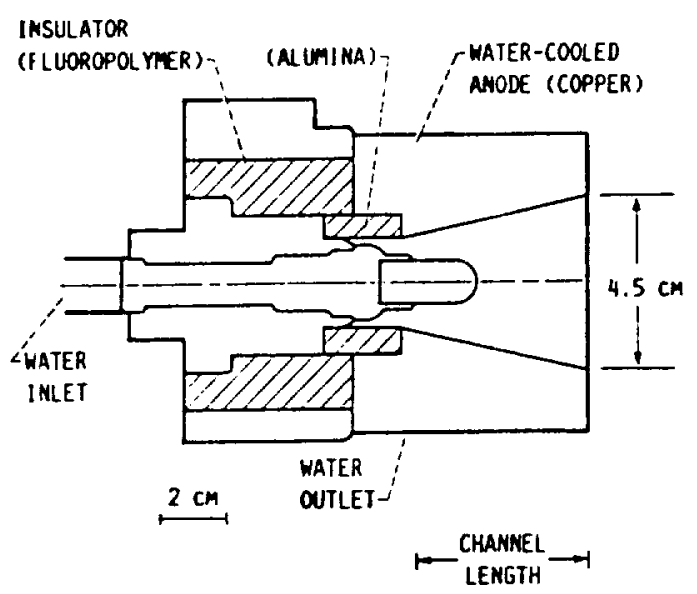

Geometry B

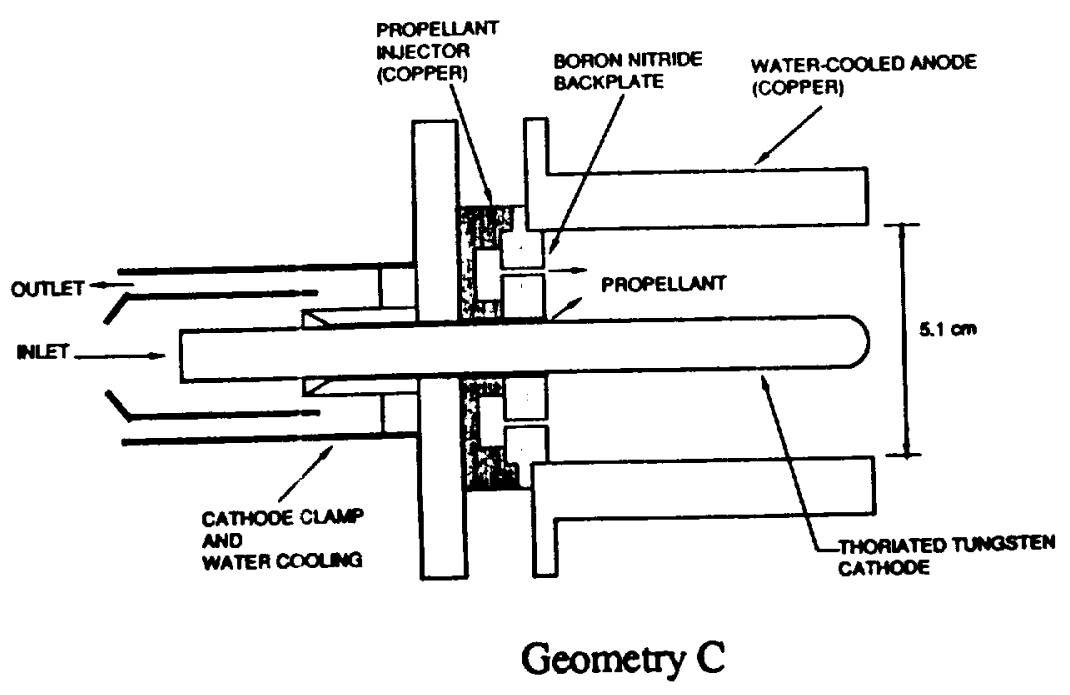

Figure 2: MPD Thruster Geometries 


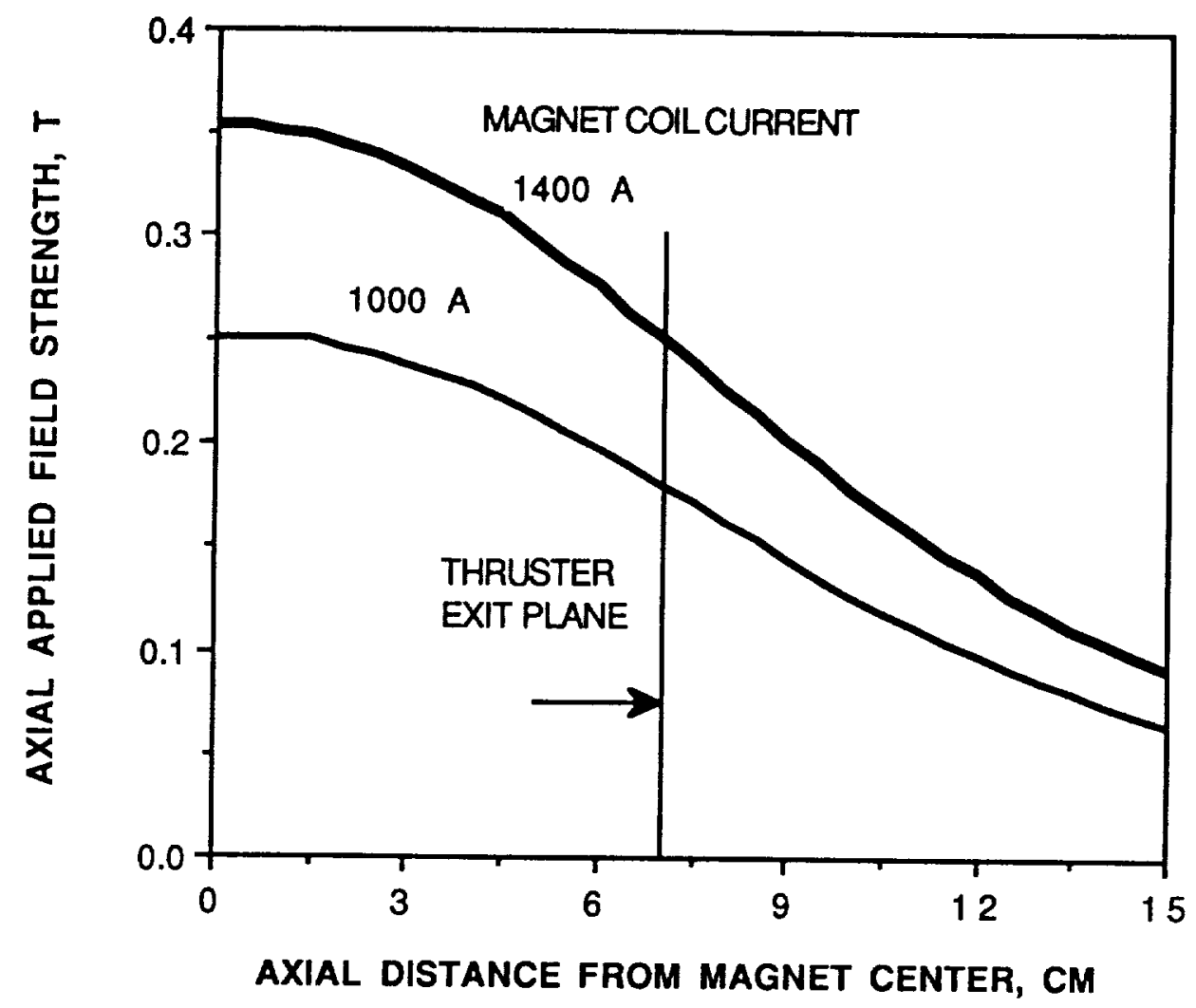

Figure 3: Axial applied-field strength vs. axial position along centerline.

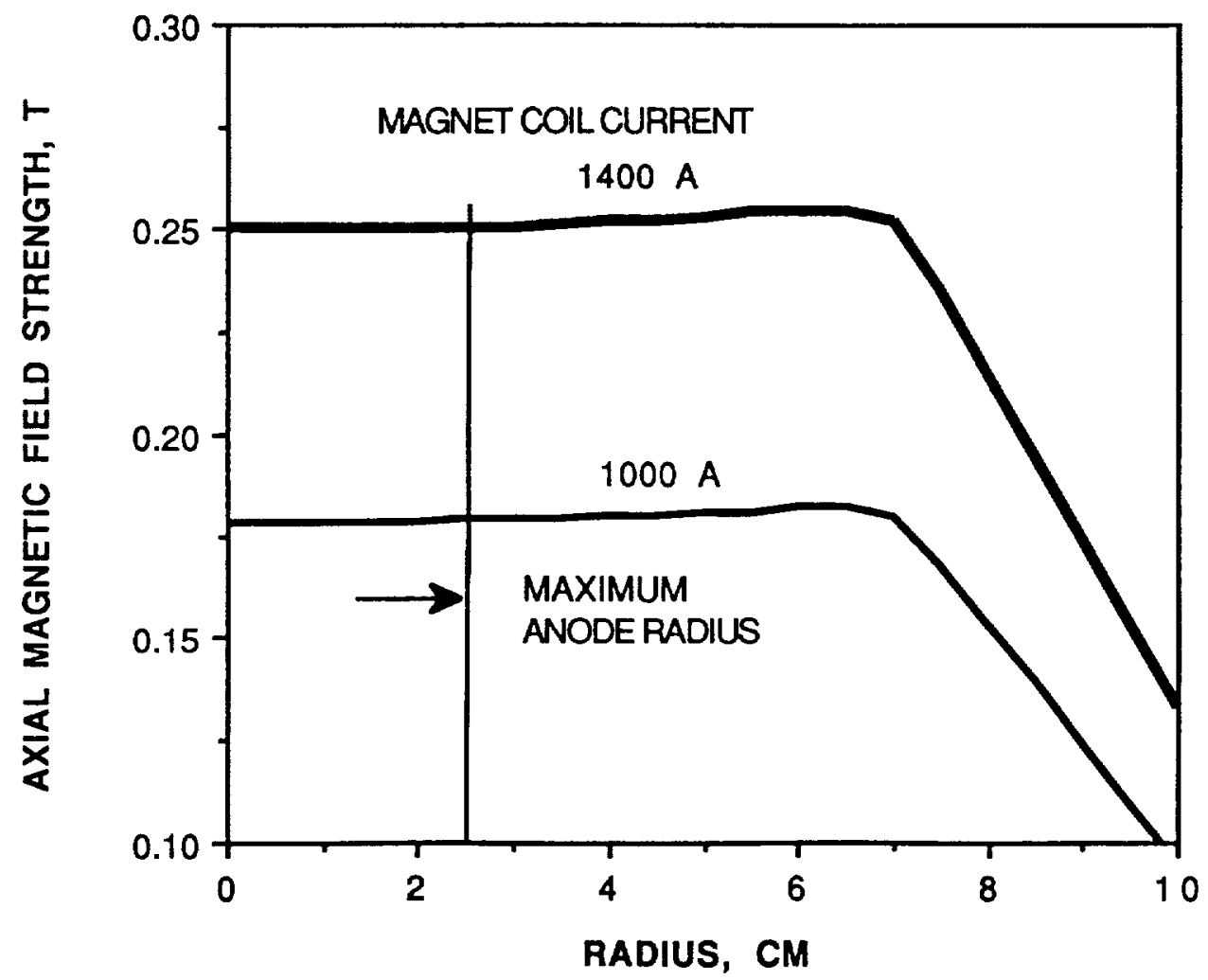

Figure 4: Axial applied-field strength at thruster exit plane vs. radius. 


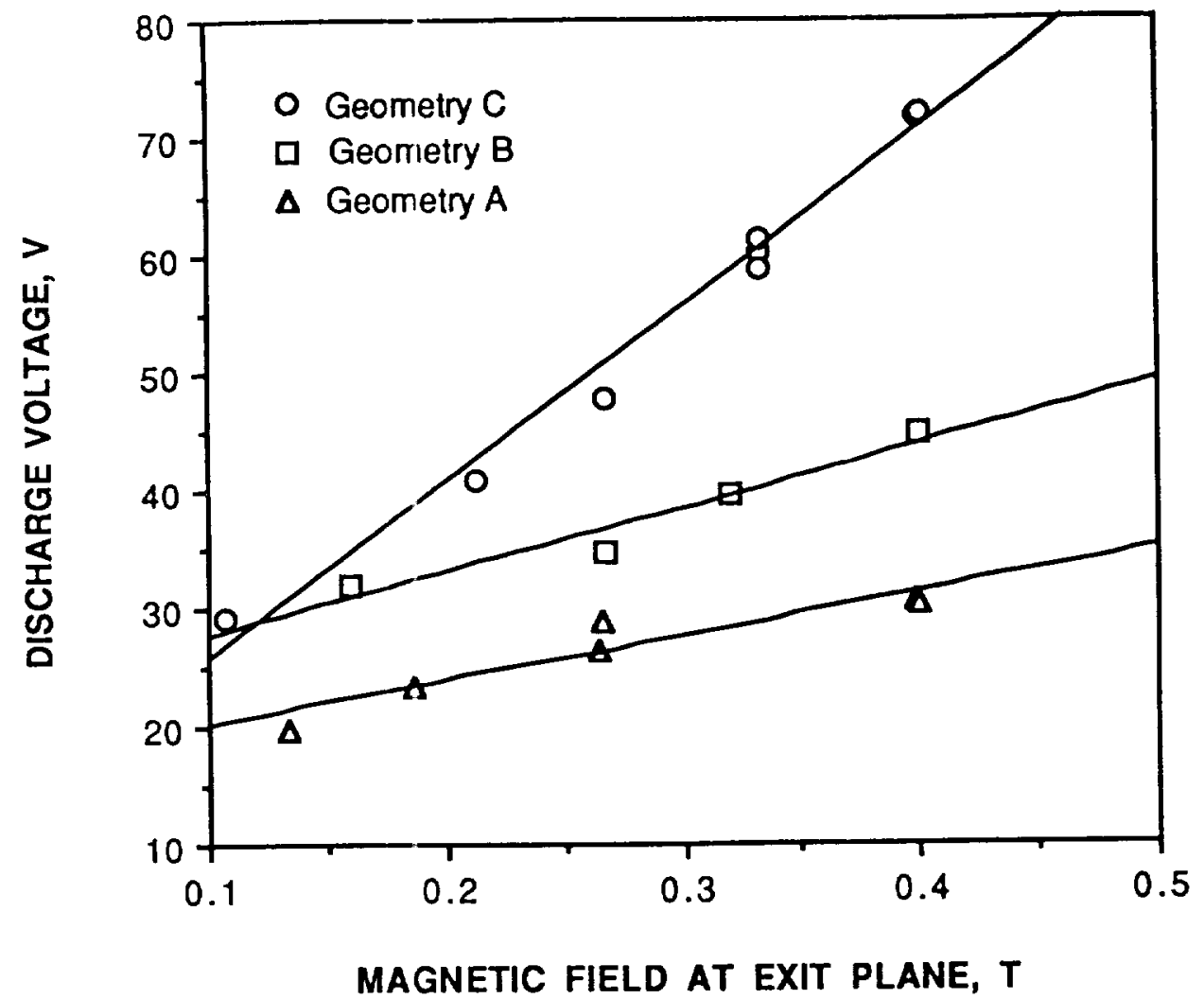

Figure 5: Discharge voltage vs. magnetic field strength at thruster exit plane centerline $\left(J_{d}=1000 A, \dot{m}=0.14 g / s\right)$.

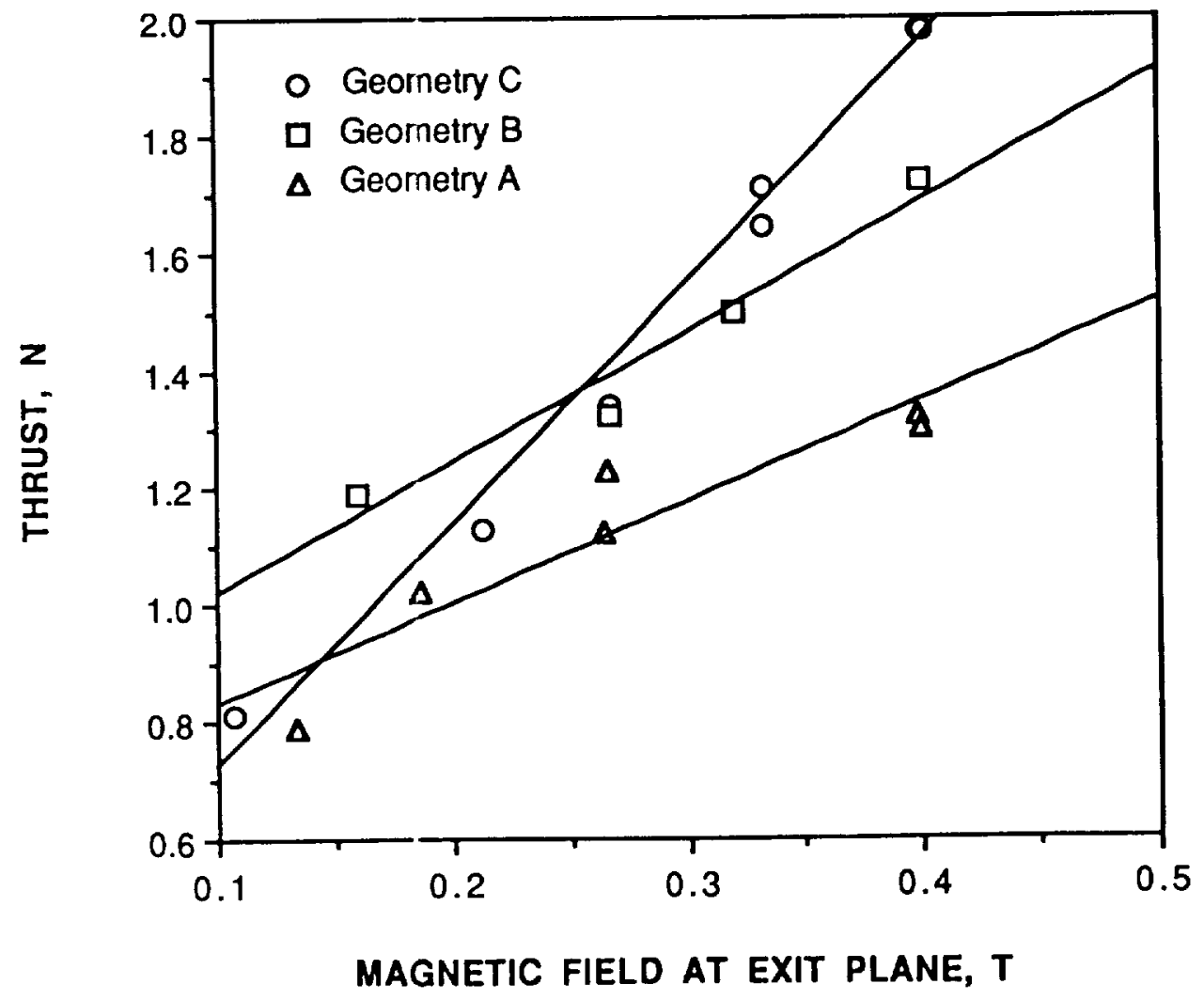

Figure 6: Thrust vs. applied magnetic field strength at exit plane centerline $\left(\mathrm{J}_{\mathrm{d}}=1000 \mathrm{~A}, \dot{\mathrm{m}}=\mathbf{0 . 1 4} \mathrm{g} / \mathrm{s}\right)$. 


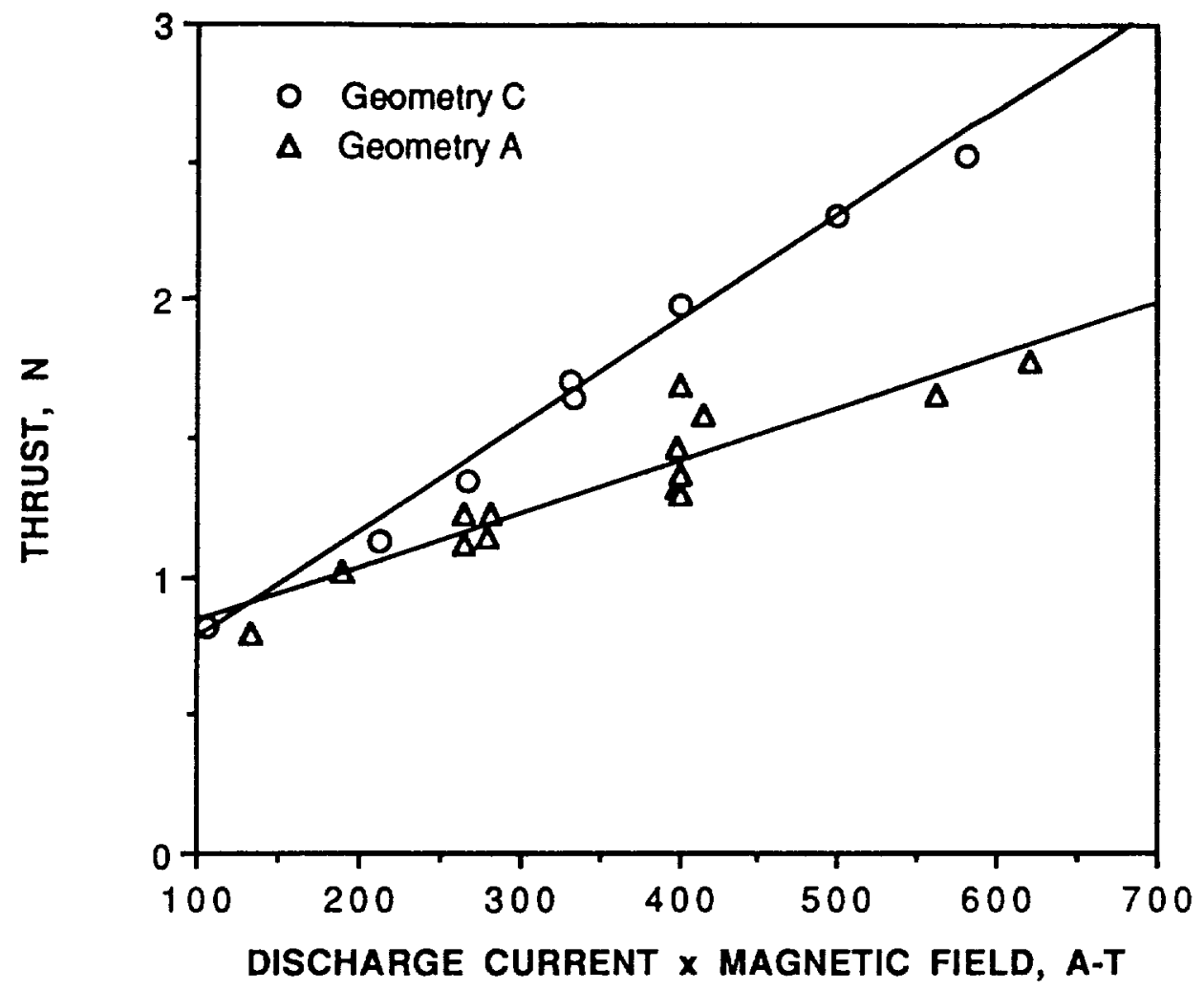

Figure 7: Thrust vs. the product of the dischage current and the magnetic field strength $\left(J_{d}=1000 A, \dot{m}=0.14 \mathrm{~g} / \mathrm{s}\right)$.

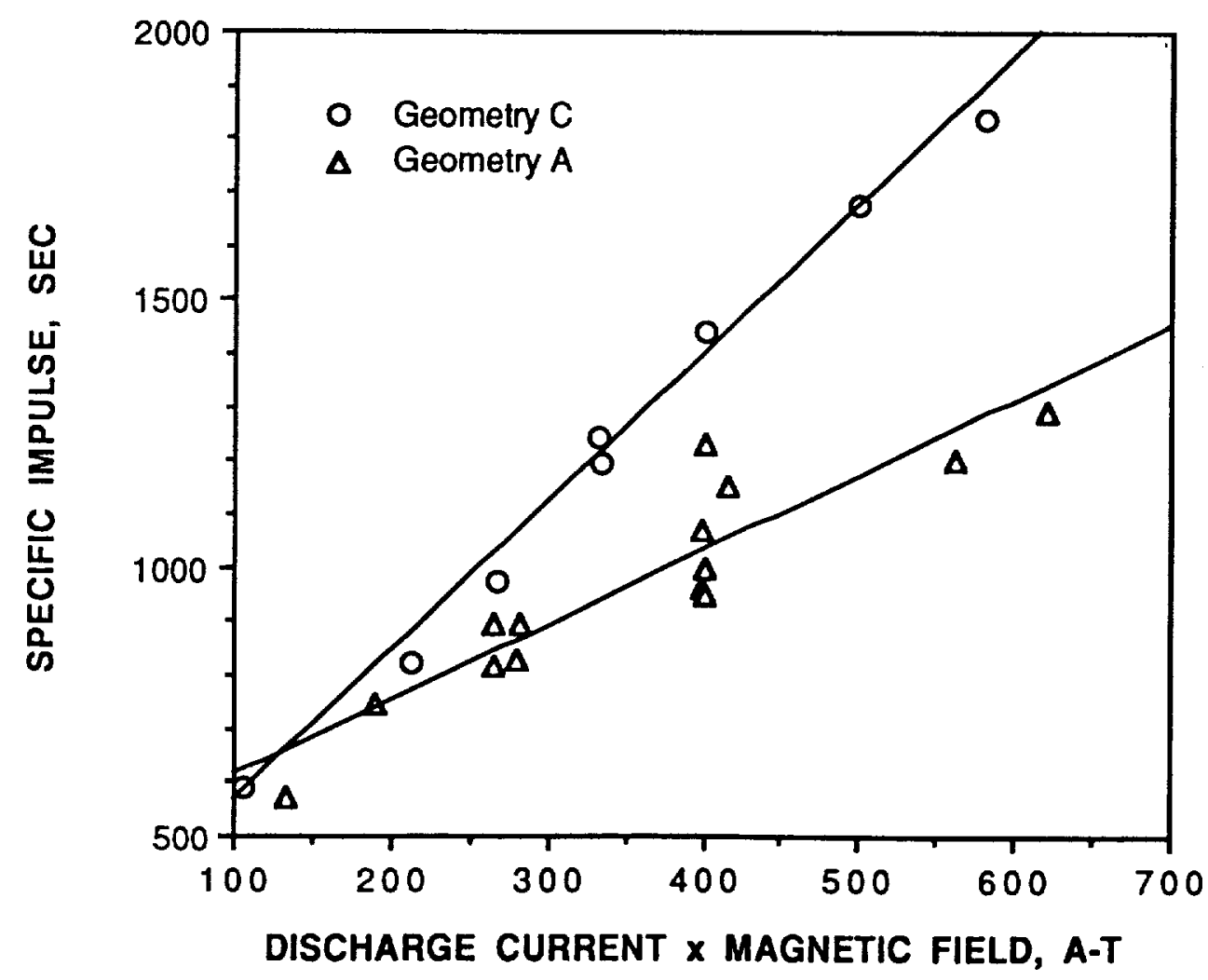

Figure 8: Specific Impulse vs. the product of the discharge current and the magnetic field strength $\left(J_{d}=1000 A, \dot{m}=0.14 \mathrm{~g} / \mathrm{s}\right)$. 


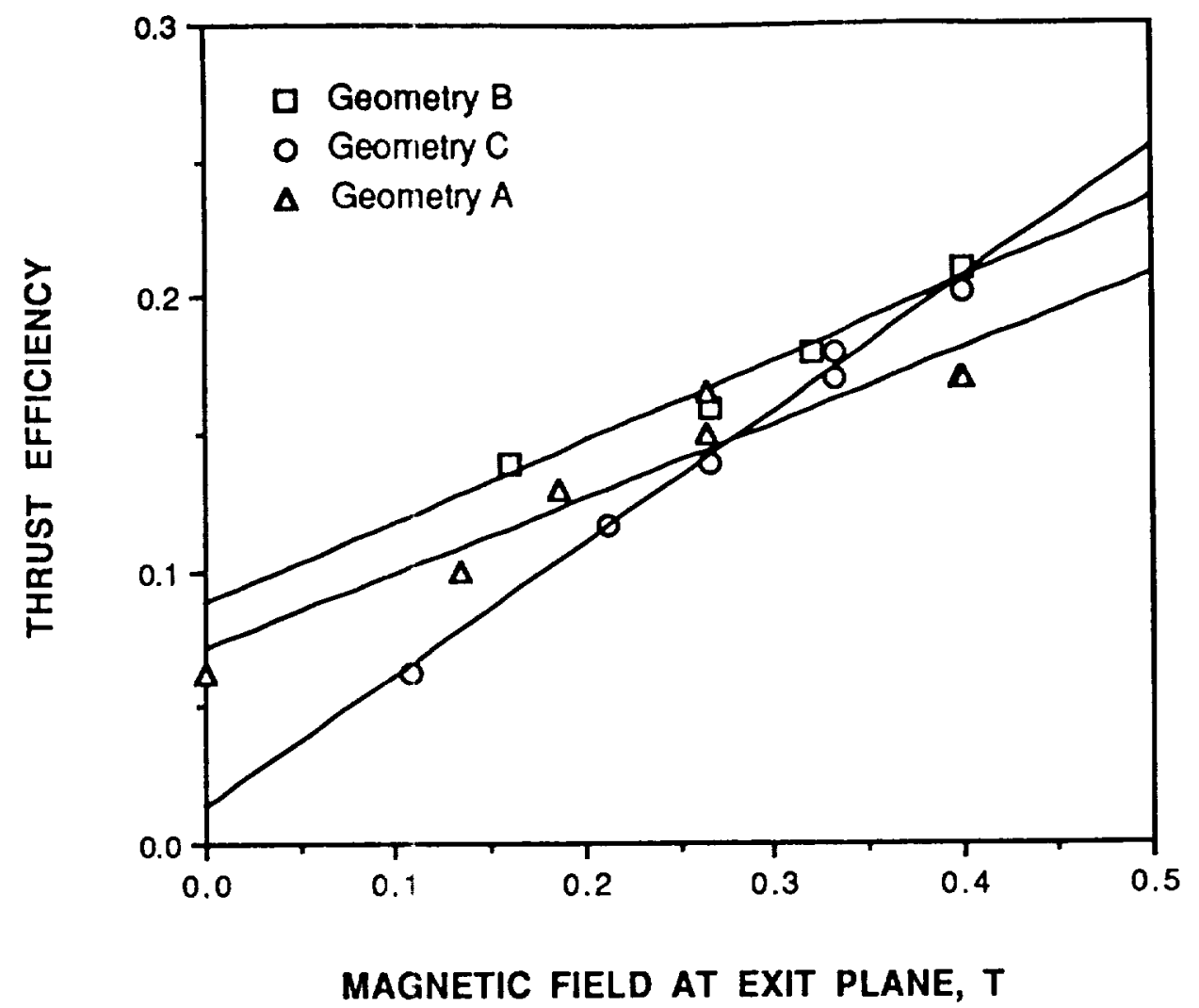

Figure 9: Thrust efficiency vs. magnetic field strength at the thruster exit plane centerline $\left(J_{d}=1000 \mathrm{~A}, \dot{\mathrm{m}}=0.14 \mathrm{~g} / \mathrm{s}\right)$.

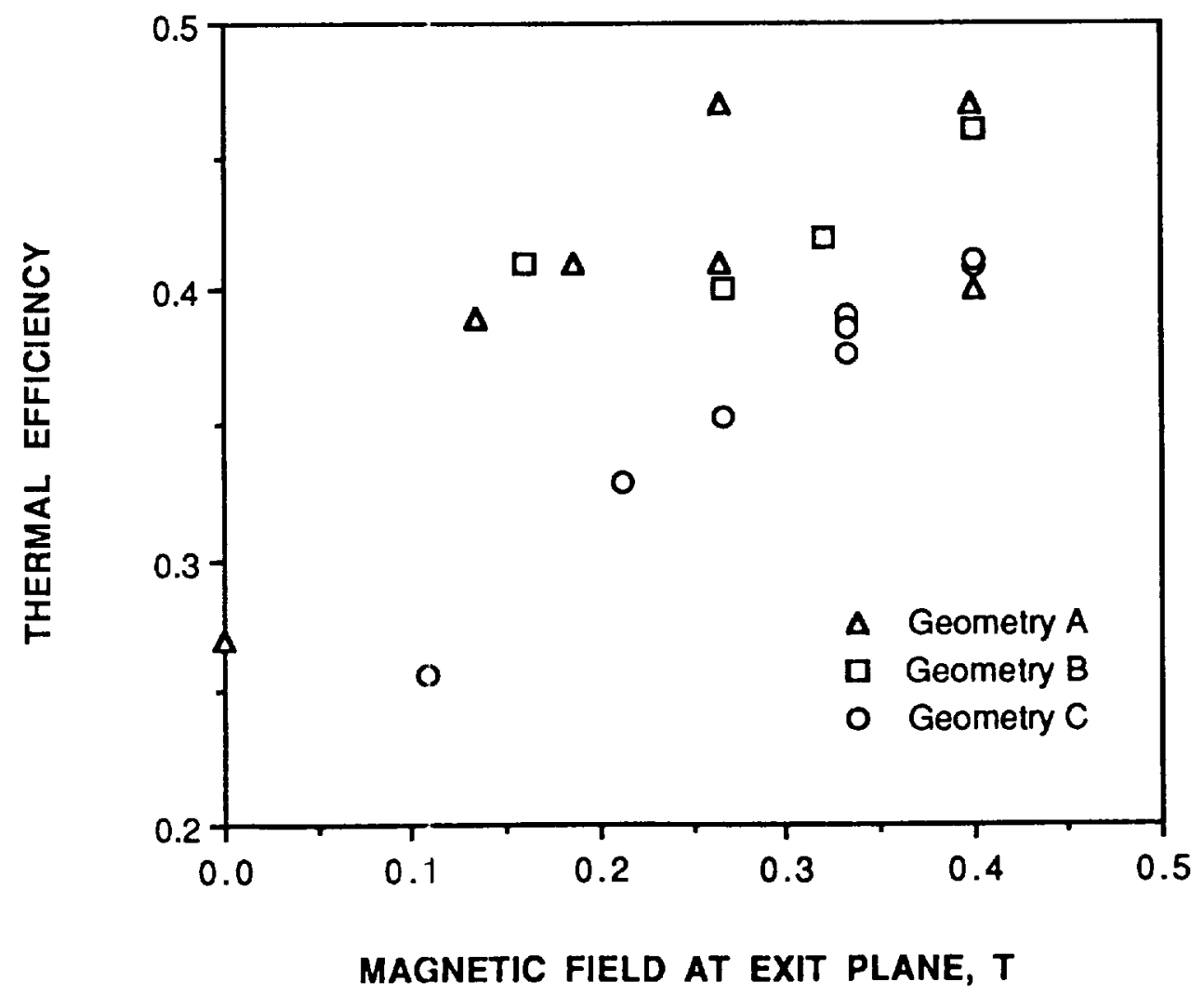

Figure 10: Thermal efficiency vs. magnetic field strength at the thruster exit plane centerline $\left(\mathrm{J}_{d}=1000 \mathrm{~A}, \dot{\mathrm{m}}=0.14 \mathrm{~g} / \mathrm{s}\right)$. 


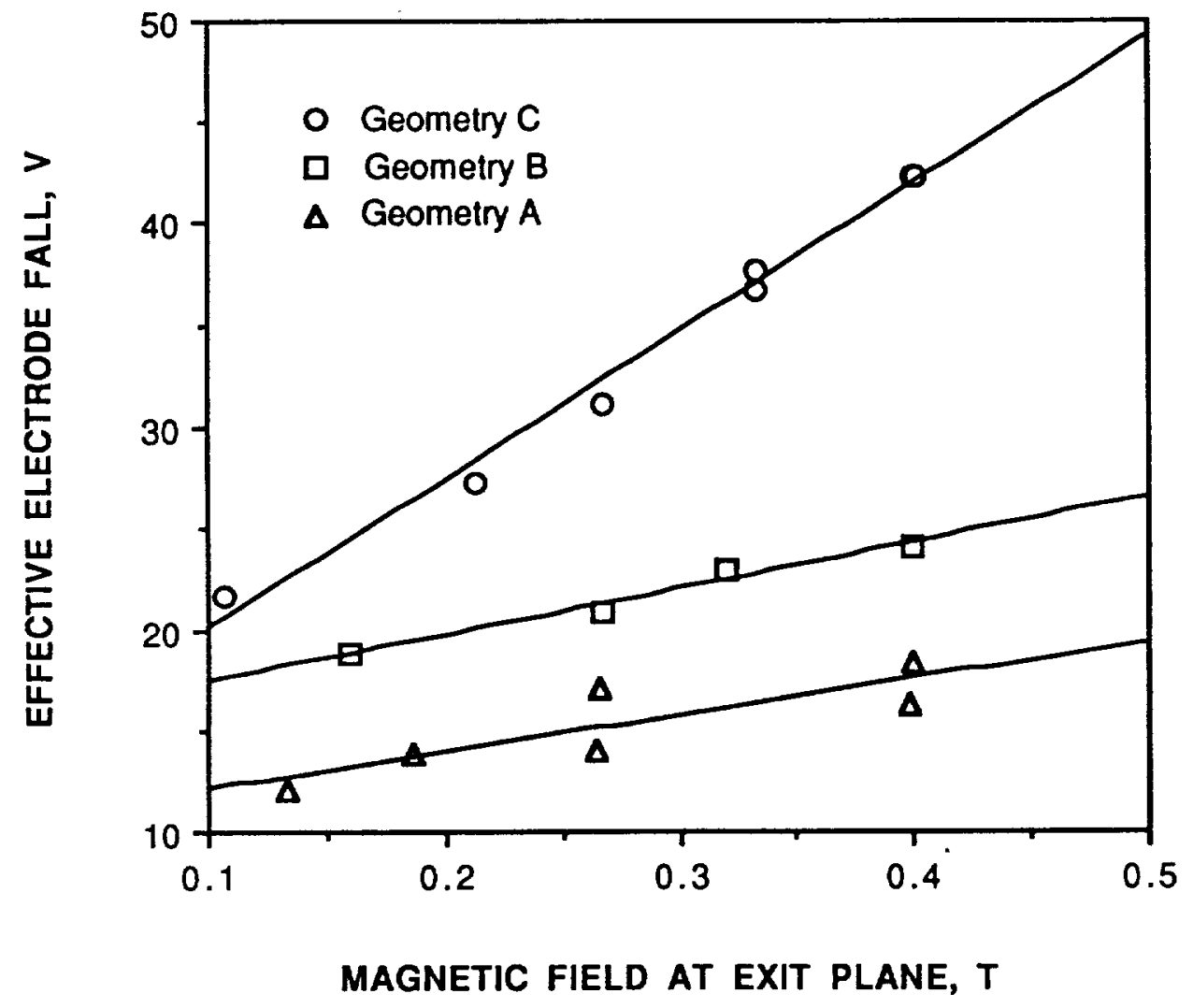

Figure 11: Effective electrode fall vs. magnetic field strength at the thruster exit plane centerline $\left(J_{d}=1000 \mathrm{~A}, \dot{\mathrm{m}}=0.14 \mathrm{~g} / \mathrm{s}\right)$. 


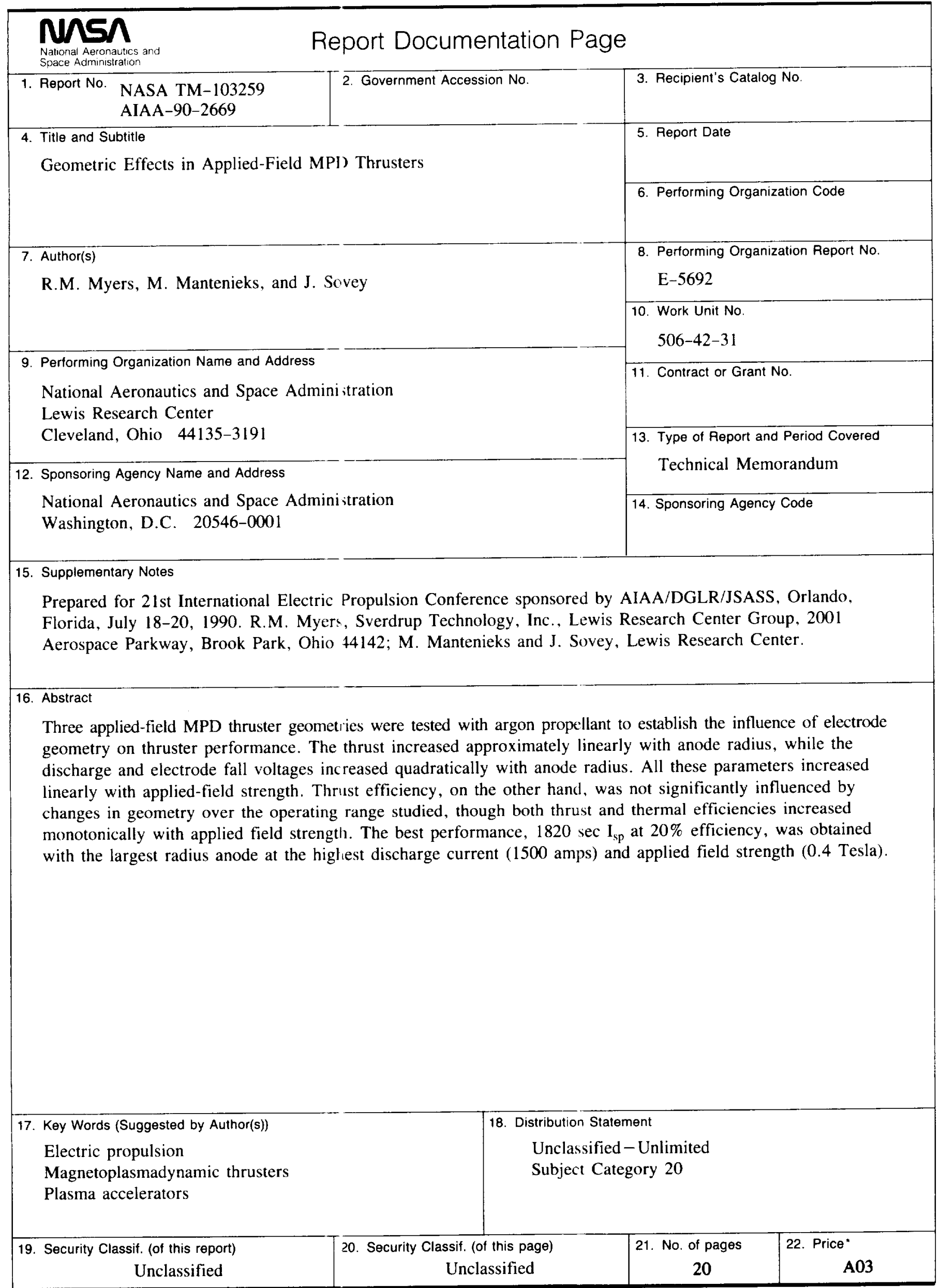




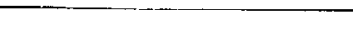

